\title{
Convergent Normal Forms of Symmetric Dynamical Systems
}

\author{
G. Cicogna \\ Dipartimento di Fisica, Università di Pisa, P.za Torricelli 2, I-56126, Pisa, Italy \\ E-mail : cicogna@ipifidpt.difi.unipi.it
}

\begin{abstract}
.
It is shown that the presence of Lie-point-symmetries of (non-Hamiltonian) dynamical systems can ensure the convergence of the coordinate transformations which take the dynamical sytem (or vector field) into Poincaré-Dulac normal form.
\end{abstract}




\section{Introduction}

A well known and interesting procedure, going back to the classical work of Poincaré, for investigating analytic vector fields $(\mathrm{VF}) X_{f}$

$$
X_{f} \equiv \sum_{i=1}^{n} f_{i}(u) \frac{\partial}{\partial u_{i}} \equiv f \cdot \nabla \quad\left(u \in \mathbf{R}^{n}\right)
$$

or the associated dynamical sytems (DS)

$$
\frac{\mathrm{d} u}{\mathrm{~d} t}=f(u) \quad u=u(t)
$$

in a neighbourhood of a stationary point, is that of introducing some new coordinates in which the given VF takes its "simplest" form, i.e. the normal form (NF) (in the sense of Poincaré-Dulac [1-4]). These coordinate transformations are usually performed by means of recursive techniques: in general the normalizing transformations (NT) are actually purely formal transformations, and only very special conditions can ensure their convergence and the (local) analyticity of the NF [1-4]. In the investigation of these problems, a relevant role can be played by the presence of some symmetry property [5,6] (see also [7]) of the VF $X_{f}$, i.e. by the presence of some $\mathrm{VF} X_{g}$

$$
X_{g} \equiv \sum_{i=1}^{n} g_{i}(u) \frac{\partial}{\partial u_{i}} \equiv g \cdot \nabla
$$

such that

$$
\left[X_{f}, X_{g}\right]=0
$$

In terms of the DS (2), the symmetry VF $X_{g}$ provides the Lie generator of a (possibly non-linear) Lie-pointsymmetry of the DS, and this can be conveniently expressed in the form of the Lie-Poisson bracket

$$
\{f, g\}_{i} \equiv(f \cdot \nabla) g_{i}-(g \cdot \nabla) f_{i}=0 \quad(i=1, \ldots, n)
$$

In this context, Bruno and Walcher [8] showed that the existence of an analytic symmetry for a 2-dimensional DS is enough to ensure convergence of the NT; in [9] the convergence is obtained also for DS of dimension $n>2$, combining the existence of symmetries with other conditions involving also the constants of motion of the DS; in $[10,11]$ the role of symmetries is investigated in view of the problem of linearizing the DS. In this paper, we will discuss some generalizations and some results along the same lines. 


\section{Preliminary results}

We will freely use $f$ both to denote the VF $X_{f}$ (1) and to refer to the DS (2); let us introduce the notation

$$
f(u)=A u+F(u)
$$

where $f$ is assumed to be analytic in a neighbourhood of the stationary point $u_{0}=0$, and its linear part $A=(\nabla f)(0)$ a semisimple (and not zero) matrix. The NF of $f$ will be written

$$
\widehat{f}(u)=A u+\widehat{F}(u)
$$

(the notation $\hat{\imath}$ will be always reserved to NF; there is no danger of confusion if $u$ is used to denote also the "new" coordinates), and $\widehat{F}(u)$ contains the "resonant terms" with respect to $A$, i.e. the terms such that

$$
\widehat{F}(u) \in \operatorname{Ker}(\mathcal{A})
$$

where $\mathcal{A}$ is the "homological operator" defined by

$$
\mathcal{A}(h)=\{A u, h\}
$$

This fact can be conveniently stated in the form:

Proposition 1. Every NF $\widehat{f}$ admits the linear symmetry $g_{A} \equiv A u$.

Let us recall some well known and useful facts.

Lemma 1. Every symmetry

$$
g(u)=B u+G(u)
$$

of a NF $\widehat{f}$ is also a symmetry of the linear part $A u$ of $\widehat{f}$. The analogous result is true for the constants of motion of the DS (6): i.e., if a scalar function $\mu=\mu(u)$ is such that $\widehat{f} \cdot \nabla \mu=0$ then also $A u \cdot \nabla \mu=0$.

Denoting by $\mathcal{G}_{f}$ and $\mathcal{I}_{f}$ the set of the symmetries and respectively of the constants of motion of $f$, we can then write

$$
\mathcal{G}_{\widehat{f}} \subseteq \mathcal{G}_{A u} \quad \text { and } \quad \mathcal{I}_{\widehat{f}} \subseteq \mathcal{I}_{A u}
$$

This is true not only for analytic quantities, but also for quantities expressed by means of formal power series.

Lemma 2. Given the matrix $A$, the most general NF has the form

$$
\widehat{F}(u)=\sum_{j} \mu_{j}(u) M_{j} u \quad \text { with } \quad \mu_{j}(u) \in \mathcal{I}_{A u} \quad \text { and } \quad\left[M_{j}, A\right]=0
$$

where the sum is extended to a set of linearly independent matrices $M_{j}$ (the set of these matrices clearly includes $A$ ).

Lemma 3. If $f$ admits a linear symmetry $g_{B}=B u$, then the NF $\widehat{f}$ also admits this symmetry. If $f$ admits a (possibly formal) symmetry $g=B u+G(u)$ and $B$ is semisimple, then $B u$ is a symmetry of the NF $\widehat{f}$, or - in other words $-\widehat{F}$ is a $\mathrm{NF}$ also with respect to $B$, i.e. $\widehat{F} \in \operatorname{Ker}(\mathcal{A}) \cap \operatorname{Ker}(\mathcal{B})$. 
The proofs of these Lemmas are well known and can be found, e.g., in [7,12-14]. Let us also recall the basic conditions, found by Bruno [1,2], and called Condition $\omega$ and Condition A, which ensure the convergence of the NT of a given DS. Denoting by $\lambda_{1}, \ldots, \lambda_{n}$ the eigenvalues of the matrix $A$, then the first condition is

Condition $\omega$ : let $\omega_{k}=\min |(q, \lambda)|$ for all positive integers $q_{i}$ such that $\sum_{i=1}^{n} q_{i}<2^{k}$ and $(q, \lambda)=\sum_{i} q_{i} \lambda_{i} \neq 0$ : then

$$
\sum_{k=1}^{\infty} 2^{-k} \ln \omega_{k}^{-1}<\infty
$$

This is a very weak condition, and we explicitly assume from now on that it is always satisfied. The other one, instead, is a quite strong restriction on the form of the NF. To state this condition in its simplest form, let us assume for a moment that there is a straight line through the origin in the complex plane which contains all the eigenvalues $\lambda_{i}$ of $A$, and that there are eigenvalues lying on both sides of this line with respect to the origin. Then the condition reads

Condition A: there is a coordinate transformation changing $f$ to $\widehat{f}$, where $\widehat{f}$ has the form

$$
\widehat{f}=A u+\alpha(u) A u
$$

and $\alpha(u)$ is some scalar-valued power series (with $\alpha(0)=0)$.

In the case there is no line in the complex plane which satisfies the above property, then Condition A should be modified [1] (or even weakened: for instance, if there is a straight line through the origin such that all the $\lambda_{i}$ lie on the same side of this line, then the eigenvalues belong to a Poincaré domain $[1,3]$ and the convergence is guaranteed without any other condition); but in all the applications below, where in particular only linear NF will be ultimately concerned, the above formulation of Condition A is enough to cover all the cases to be considered, and we can say $[1,2]$ that there is a convergent NT if the above conditions are satisfied. Clearly, here and in the following, "convergence" stands for "convergence in some open neighbourhood of $u_{0}=0$ ".

\section{Symmetries and convergence of the NT: a general result}

Let us finally state the first result of this paper. It can be noted that quite strong assumptions are needed; but it is known, on the other hand, that the convergence of the NT is quite "exceptional". The examples given below will show how, thanks to additional symmetry properties, these assumptions can happen to be verified. Let us remark that obviously, for any constant $c$, then $c f$ is a (trivial) symmetry of $f$; therefore, it is understood that when we assume the existence of some symmetry of $f$ we will always refer to nontrivial symmetries, i.e. to symmetries $g \neq c f$.

Theorem 1. Given the analytic VF $f$, let us write its NF, according to Lemma 2, in the form

$$
\widehat{f}=A u+\alpha(u) A u+\sum^{\prime} \mu_{j}(u) M_{j} u \equiv A u+\alpha(u) A u+\widehat{F}_{1}(u)
$$

where (here and in the following) $\sum^{\prime}$ is the sum extended to the matrices $M_{j} \neq A$. Assume $\widehat{F}_{1}(u) \neq 0$ (otherwise Condition A is sufficient of ensure convergence of the NT), and: 
a) assume that $f$ admits an analytic symmetry

$$
g=B u+G(u) \quad \text { such that } \quad B=a A
$$

where $a$ is a (possibly vanishing) constant;

b) assume that the equation

$$
\left\{\widehat{F}_{1}, S\right\}=0
$$

for the unknown

$$
S=S(u)=\sum^{\prime} \nu_{j}(u) M_{j} u \quad \text { with } \quad \nu_{j}(u) \in \mathcal{I}_{A u} \quad \text { and } \quad \nu_{j}(0)=0
$$

has only the trivial solution

$$
S=c \widehat{F}_{1}(u) \quad(c=\text { constant })
$$

Then $f$ can be put into NF by means of a convergent NT.

Proof. First of all, if $a=0$ in assumption a), one can consider, instead of $g$, the symmetry $g^{\prime}=f+g$ having linear part $A u$; it is then not restrictive to assume $a=1$, i.e. $B=A$. Once $f$ is put into NF $\widehat{f}$, the symmetry $g$ will become a (possibly formal) symmetry $\widetilde{g}$

$$
\widetilde{g}=A u+\beta(u) A u+\sum^{\prime} \nu_{j}(u) M_{j} u \quad \text { with } \quad \beta(u), \nu_{j}(u) \in \mathcal{I}_{A u}
$$

this is indeed the most general symmetry of a NF, thanks to Lemma 1 . The symmetry condition $\{f, g\}=0$ in the new coordinates reads $\{\widehat{f}, \widetilde{g}\}=0$; evaluating term by term this bracket, one is left with

$$
\left\{\alpha A u, \sum_{j}^{\prime} \nu_{j} M_{j} u\right\}+\left\{\sum_{j}^{\prime} \mu_{j} M_{j} u, \beta A u\right\}+\left\{\sum_{j}^{\prime} \mu_{j} M_{j} u, \sum_{k}^{\prime} \nu_{k} M_{k} u\right\}=0
$$

or

$$
\left(\sum_{j}^{\prime} \mu_{j}(u) M_{j} u \cdot \nabla \beta-\sum_{j}^{\prime} \nu_{j}(u) M_{j} u \cdot \nabla \alpha\right) A u+\left\{\sum_{j}^{\prime} \mu_{j}(u) M_{j} u, \sum_{k}^{\prime} \nu_{k}(u) M_{k} u\right\}=0
$$

All other terms in fact vanish thanks to Proposition 1 and Lemmas 2 and 3. Now, in eq.(17), the bracket $\{\cdot, \cdot\}$ produces, through the matrix commutators $\left[M_{j}, M_{k}\right]$, only terms proportional to $M_{j} u$ (and not to $A u$ : this can be easily seen in a basis in which $A$ is diagonal), therefore the terms appearing into the ( ) and the bracket $\{\cdot, \cdot\}$ are both zero. The last bracket has just the form $\left\{\widehat{F}_{1}, S\right\}$, and therefore assumption $b$ ) gives $S=c \widehat{F}_{1}$, i.e. $\nu_{j}(u)=c \mu_{j}(u)$. From the vanishing of the first $(\quad)$ in (17), and using again assumption $b$ ), one obtains similarly $\beta(u)=c \alpha(u)$, and then either $\widetilde{g}=c \widehat{f}$, which is impossible because $g \neq c f$, or

$$
\widetilde{g}(u)=A u
$$

This means that the transformation which puts $f(u)$ into $\widehat{f}(u)$ transforms $g(u)=A u+G(u)$ into $\widetilde{g}(u)=A u$, therefore the symmetry $g(u)$ satisfies Condition A and there is convergent transformation which puts $g(u)$ into NF. Under this convergent transformation $f(u)$ is transformed into $\mathrm{NF} \widehat{f}$, as a consequence of the last part of Lemma 3.

Remark 1. One can see that the assumption b) of Theorem 1 is equivalent to the assumption that the NF $\widehat{f}$ admits only linear symmetries $L u$. For a practical point of view (see the Examples below), it is quite simpler to verify the property b). 
Remark 2. In the particular case that $\widehat{F}_{1}(u)$ has the form

$$
\widehat{F}_{1}(u)=\mu(u) M u
$$

(with $M \neq A$ ), then assumption $b$ ) is actually equivalent to the very simple following one: there are no common (analytic, formal or fractional) constants of motion of the two linear problems

$$
\dot{u}=A u \quad \text { and } \quad \dot{u}=M u
$$

Indeed, assume there is some $\kappa=\kappa(u) \in \mathcal{I}_{A u} \cap \mathcal{I}_{M u}$, then $S=\kappa(u) A u \neq c \widehat{F}_{1}$ would satisfy $\left\{\widehat{F}_{1}, S\right\}=0$; notice, incidentally, that one would also get in this case $\kappa(u) \in \mathcal{I}_{\widehat{f}}$. The converse is easily obtained by explicit calculations. This case has been already considered in [9]; the result for 2-dimensional DS in [8] can be viewed as a particular case of this (see [9] for details).

The apparent difficulty in the application to concrete cases of the above results is that, in general, one does not known - a priori - the NF, and then it seems to be impossible to check if the assumptions of Theorem 1 (or even Condition A) are verified by the NF. However, as the foregoing Examples will show, other symmetry properties of the VF may provide, once again, the decisive help on this point.

Example 1. Consider a 3-dimensional analytic DS

$$
\dot{u}=f(u)=A u+F(u) \quad \text { with } \quad A=\operatorname{diag}(1,1,-2)
$$

with $u \equiv(x, y, z) \in \mathbf{R}^{3}$ and assume that $f(u)$ possesses the linear $S_{2}$ symmetry generated by $L u \cdot \nabla$ where

$$
L=\left(\begin{array}{ccc}
0 & 1 & 0 \\
-1 & 0 & 0 \\
0 & 0 & 0
\end{array}\right)
$$

i.e. $f$ is "equivariant" under rotations in the plane $(x, y)$. Putting $r^{2}=x^{2}+y^{2}$, this implies that $F(u)$ must be written in the form

$$
F(u)=\varphi_{0}\left(r^{2}, z\right) A u+\varphi_{1}\left(r^{2}, z\right) I u+\varphi_{2}\left(r^{2}, z\right) L u
$$

where $I$ is the identity matrix in $\mathbf{R}^{3}$. If we now choose, for instance,

$$
\varphi_{0}=0, \quad \varphi_{1}=a_{1} r^{2} z+a_{2} z^{3}, \quad \varphi_{2}=b \varphi_{1}
$$

where $a_{1}, a_{2}, b$ are constants $\neq 0$, then the DS admits also the non-linear symmetry

$$
G(u)=r^{2} z(I+b L) u
$$

Notice that the assumption $a_{2} \neq 0$ ensures that this DS is not a NF, and that the above symmetry is not trivial. Then assumption a) of Theorem 1 is satisfied. Now, the NF of the above DS (21-24) must be of the form

$$
\widehat{f}=A u+\alpha\left(r^{2} z\right) A u+\mu_{1}\left(r^{2} z\right) I u+\mu_{2}\left(r^{2} z\right) L u
$$

where $\alpha, \mu_{1}, \mu_{2}$ depend only on $\kappa=r^{2} z$, as a consequence of Lemma 3 (i.e., the equivariance under $S O_{2}$ is preserved), and of Lemma 2 (the resonance condition). We have to look for the solutions $S$ of the equation $\left\{\widehat{F}_{1}, S\right\}=0$, where the unknown $S$ can be written

$$
S=\nu_{1}(u) I u+\nu_{2}(u) L u \quad \text { with } \quad \nu_{1}(u), \nu_{2}(u) \in \mathcal{I}_{A u}
$$


(it is easy to see that no other matrices can appear in the r.h.s. of (26-27)), and where $\nu_{1}, \nu_{2}$ must be functions only of the two functionally independent quantities $x^{2} z, x y z \in \mathcal{I}_{A u}$. The condition $\left\{\widehat{F}_{1}, S\right\}=0$ gives the first-order system of linear partial differential equations

$$
\begin{aligned}
& \left(\mu_{1} u+\mu_{2} L u\right) \cdot \nabla \nu_{1}=\nu_{1} u \cdot \nabla \mu_{1} \\
& \left(\mu_{1} u+\mu_{2} L u\right) \cdot \nabla \nu_{2}=\nu_{1} u \cdot \nabla \mu_{2}
\end{aligned}
$$

Observing that $L u \cdot \nabla \mu_{1}=0$ and $A u \cdot \nabla \mu_{1}=0=u \cdot \nabla \mu_{1}-3 z \partial_{z} \mu_{1}$ and the same for $\nu_{1}$, i.e. $u \cdot \nabla \nu_{1}=$ $3 z \partial_{z} \nu_{1}$, one gets from the first of the (28) the characteristic equation for the unknown $\zeta=\zeta(u)$ defined by $\nu_{1}(u)=\zeta(u) \mu_{1}(u)$

$$
\frac{\mathrm{d} x}{y}=-\frac{\mathrm{d} y}{x}=\frac{\mu_{2}}{\mu_{1}} \frac{\mathrm{d} z}{3 z}
$$

which shows that $\zeta$ must be a function of $r^{2}$ and of some other variable of the form $v=\operatorname{arctg}(y / x)+Z(z)$. On the other hand, $\mu_{1}$ is a function of $\kappa=r^{2} z$, and $\nu_{1}$ is a function of the quantities $x^{2} z, x y z \in \mathcal{I}_{A}$ (possibly also of $x / y$, of course, the only requirement is that $\mu_{1}, \nu_{1}$ are power series in $\left.x, y, z\right)$; it is then easy to see that $\zeta=$ const, i.e. that $\nu_{1}=c \mu_{1}$. Proceeding in the same way for the other equation in (28), one can conclude that

$$
S=c \widehat{F}_{1}
$$

and then also the assumption b) in Theorem 1 is satisfied, and therefore there is a convergent NT.

Notice that it was essential in the calculations for the example above that $\mu_{1}$ and $\mu_{2}$ are both $\neq 0$, and this is in fact guaranteed by the normalizing procedure: indeed, at the lowest order, the resonant terms $r^{2} z I u$ and $r^{2} z L u$ are orthogonal to $z^{3} I u$ and $z^{3} L u$ (with respect to standard scalar product $[3,12,14]$ introduced in the vector space of homogeneous polynomials, where the homological operator $\mathcal{A}$ is defined), then at the first step of the normalization procedure the resonant terms are not changed, i.e. one has $\mu_{1}=a_{1} r^{2} z+\ldots$ and $\mu_{2}=a_{1} b r^{2} z+\ldots ;$ and then - at any further step of the iteration - the lower order terms are not altered.

\section{Symmetries and convergence of the NT: a special case}

Let us now come back to the special case that $\widehat{F}_{1}(u)$ can be written in the form

$$
\widehat{F}_{1}(u)=\mu(u) M u
$$

In Remark 2 we have seen that assumption $b$ ) of Theorem 1 can be replaced by the requirement that there are no simultaneous constants of motion of $A u$ and $M u$. Assume now that, as in Example 1, the DS admits not only the non-linear symmetry $g(u)$ (assumption a) of Theorem 1), but also a symmetry generated by some linear VF $L u \cdot \nabla$, and that $g(u)$ satisfies

$$
\{g, L u\}=0
$$

In the NT the symmetry is conserved step by step [14], therefore $g(u)$ also will be transformed, once $f$ is in NF $\widehat{f}$, into some $\widetilde{g}(u)$ which is symmetric under $L u:\{\widetilde{g}, L u\}=0$. From this remark we see that it is sufficient to look for the common constants of motion of $A u$ and $M u$ only in the set of those scalar functions $\kappa=\kappa(u)$ 
which are left invariant by $L u$; in other words, we can conclude that if the set of these simultaneously invariant functions contains only trivially constant numbers, i.e.

$$
\mathcal{I}_{A u} \cap \mathcal{I}_{M u} \cap \mathcal{I}_{L u}=\mathbf{R}
$$

then no other non-linear symmetries are allowed, and $\widetilde{g}(u)$ becomes necessarily $\widetilde{g}(u)=A u$; then, using similar arguments as above, the convergence of the NT is guaranteed. In conclusion, we can state:

Theorem 2. Assume that $f$ admits a symmetry $g$ as in assumption a) in Theorem 1 , and also a symmetry generated by a linear VF $L u \cdot \nabla$ such that in addition (31) and (32) are satisfied. Then, if $\widehat{F}_{1}$ has the form (30), the NT is convergent. The result can be trivially extended to the case that $f$ admits an algebra of (more than one) symmetries $L_{k} u \cdot \nabla$.

Example 2. The same as Example 1, here with $b=0$ and $a_{1}, a_{2} \neq 0$. In the NF now $\mu_{1}=a_{1} r^{2} z+\ldots \neq 0$, but $\mu_{2}$ may be zero. If $\mu_{2} \neq 0$, Theorem 1 can be applied. If $\mu_{2}=0$, then $\widehat{F}_{1}$ has the form (30); on the other hand, it is clear that no $\mathrm{SO}_{2}$-invariant analytic functions are simultaneously constants of motion of $\dot{u}=A u$ and $\dot{u}=u$, then all assumptions of Theorem 2 are satisfied, and the NT is convergent.

The example given in [9] can be viewed as another example of Theorem 2, in the presence of a larger symmetry (the Lie algebra of the group $\mathrm{SO}_{3}$ ).

To conclude, it can be interesting to point out the following peculiar property of the present approach.

Remark 3. All the results in this paper are peculiar of non-Hamiltonian DS: indeed, an Hamiltonian DS never satisfies the crucial hypothesis, i.e. assumption $b)$ of Theorem 1. Let in fact $H=H(u)$, with $u \equiv(q, p) \in \mathbf{R}^{2 m}$, be an analytic Hamiltonian and

$$
\dot{u}=J \nabla H
$$

be the associated DS, where $J$ is the symplectic matrix. Writing $H=H_{0}+H_{R}$, where $H_{0}$ is the quadratic part of $H$, we have clearly $A u=J \nabla H_{0}$ and $F(u)=J \nabla H_{R}$, and the requirement that $F(u)$ is in NF, $\widehat{F}(u)=J \nabla \widehat{H}_{R}$, becomes now the requirement that $H_{0}$ is a constant of motion of $\widehat{H}_{R}$ (cf. $\left.[15,16]\right)$. Then, eq. (15) always admits nontrivial solutions of the form

$$
S=\eta\left(H_{0}\right) \widehat{F}_{1}
$$

for any (regular) function $\eta$ of $H_{0}$. 


\section{References}

[1] Bruno A.D., 1971 "Analytical form of differential equations", Trans. Moscow Math. Soc. 25, 131; and 26, 199

[2] Bruno A.D., 1989, "Local methods in nonlinear differential equations", Springer, Berlin

[3] Arnold V.I., 1988, "Geometrical methods in the theory of differential equations", Springer, Berlin

[4] Arnold V. I. and Il'yashenko Yu. S., 1988 Ordinary differential equations; in: Encyclopaedia of Mathematical Sciences - vol. I, Dynamical Systems I, (D.V. Anosov and V.I. Arnold, eds.), pp. 1-148; Springer, Berlin

[5] Olver P.J., 1986, "Applications of Lie groups to differential equations", Springer, Berlin

[6] Ovsjannikov L.V., 1962, "Group properties of differential equations", Novosibirsk; (English transl. by Bluman G.W., 1967); and 1982, "Group analysis of differential equations", Academic Press, New York

[7] Cicogna G. and Gaeta G., 1994, Journ. Phys. A: Math. Gen. 27, 461 and 7115; and 1997, IHES preprint

[8] Bruno A.D. and Walcher S., 1994, J. Math. Anal. Appl. 183, 571

[9] Cicogna G., 1995, Journ. Phys. A: Math. Gen. 28, L179, and 1996, Journ. Math. Anal. Appl. 199, 243

[10] Gaeta G. and Marmo G., 1996, Journ. Phys. A: Math. Gen. 29, 5035

[11] Bambusi D., Cicogna G., Gaeta G., and Marmo G., 1997, preprint

[12] Elphick C., Tirapegui E., Brachet M. E., Coullet P., and Iooss G., 1987, Physica D, 29, 95

[13] Walcher S., 1991, Math. Ann. 291, 293

[14] Iooss G. and Adelmeyer M., 1992, Topics in bifurcation theory and applications; World Scientific, Singapore

[15] Moser J.K., 1968, Mem. Amer. Math. Soc. 81, 1

[16] Arnold V. I., Kozlov V.V., and Neishtadt A. I., 1993, Mathematical aspects of classical and celestial mechanics; in: Encyclopaedia of Mathematical Sciences - vol. 3, Dynamical Systems III, (V.I. Arnold ed.), pp. 1-291; Springer, Berlin 